\title{
Preparation of Wood Furniture Cooling Coatings Based on Phase Change Microcapsules and its Performance Study
}

\author{
Jue Bai, ${ }^{\mathrm{a}}{ }^{Y} \mathrm{u} \mathrm{Li},{ }^{\mathrm{b}}$ Shuangshuang Jiang, ${ }^{\mathrm{c}}$ and Huiyuan Guan ${ }^{\mathrm{d}, *}$
}

\begin{abstract}
Due to the small thermal conductivity of wooden furniture, there is a better cooling effect in the initial contact with the human body during the summer. However, after long-term contact, heat cannot be exported, resulting in heat accumulation, which affects the thermal comfort. Phase change microcapsules (PCM) can be added to water-based coatings for wood furniture to achieve a lower contact temperature by using the heat absorption properties of its phase change process. Thus, it improves the thermal comfort of the human body for long-term use. In this study, the PCM were added to a water-based paint for wooden furniture. By testing the microscopic properties and thermal properties, it was found that the PCM powder could undergo phase change and absorb heat after dispersing in water-based paint. Secondly, fiberboard coated with different solid content of microencapsulated coatings was tested by homemade equipment at $27^{\circ} \mathrm{C}$ room temperature. The results showed that the addition of PCM can significantly change the contact temperature. And as the amount of microcapsules added increased, the cooling effect was more obvious. At the same time, the addition of microcapsules can cause matting of the paint film and have an effect on the hardness of the paint film. Therefore, the cooling effect can be achieved by stacking the paint film or controlling the amount of microcapsules.
\end{abstract}

DOI: 10.15376/biores.17.1.1319-1337

Keywords: Phase change microcapsules; Water-based coatings; Temperature-reducing properties; Paint film properties; Wooden furniture

Contact information: a: College of Furnishings and Industrial Design, Nanjing Forestry University, Nanjing, Jiangsu Province, China; b: College of Chemical Engineering, Nanjing Forestry University, Nanjing, Jiangsu Province, China; c: College of Chemical Engineering, Nanjing Forestry University, Nanjing, Jiangsu Province, China; $d$ : College of Furnishings and Industrial Design, Nanjing Forestry University, Nanjing, Jiangsu Province, China; *Corresponding author: nlydh2018@163.com

\section{INTRODUCTION}

With the rapid development of modern economy, people's requirements for the comfort of the indoor environment are getting higher and higher. An indoor environment that is too hot or too cold is not conducive to staff productivity and productive performance. In a moderate thermal comfort environment, office workers can easily perform their work tasks (Maula et al. 2016). From a human health perspective, indoor heat affects all aspects of human health, with the strongest evidence being the effect of high temperatures on respiratory health, diabetes, and the core symptoms of schizophrenia and dementia (Tham et al. 2020). Therefore, it is important to reduce indoor temperature and enhance human thermal comfort through furniture design and other means. Wooden furniture has also become one of the most common furniture choices due to its better temperature perception properties. However, in higher temperature indoor environments, wood furniture often 
shows better thermal comfort performance in the early stages of use. In long-term exposure, the temperature absorbed by the wood material cannot be dissipated in time, leading to heat buildup, producing a stuffy situation. And compared to other materials with faster thermal conductivity, such as metal, wood material is unable to overcome the stifling feeling in long-term contact in summer. However, these highly conductive materials are too cold at the beginning of contact and do not solve the problem of thermal comfort very well. Therefore, it is necessary to improve the thermal conductivity of the wood by modifying its surface so that it can exhibit better thermal comfort properties.

Phase change microcapsules (PCM) are a collection of materials made based on phase change principles. Microcapsules are added to coatings as fillers to achieve the effect of heat absorption and temperature reduction. When the external ambient temperature reaches the phase change temperature, the phase change material particles wrapped in the microcapsule shell material change their phase state, thus absorbing the external heat (Khadiran et al. 2016). Paraffin hydrocarbons are the most studied core materials for microcapsules, with phase change temperatures close to room temperature and large enthalpy of phase change, as well as being non-toxic, non-hazardous, and inexpensive (Huang and Wang 2012). The shell material of PCM can be divided into inorganic polymer material and organic polymer material (Peng et al. 2020). Polymethyl methacrylate (PMMA), as one of the more common shell materials for PCM, has the advantages of no formaldehyde residue, non-toxicity, and non-polluting nature. In addition, the PMMA reaction monomer is safe and widely available, and the polymerization reaction is easy to control (Sari et al. 2014). Therefore, PMMA can be used as the shell material to wrap the paraffin wax core material if it is necessary to make the system clean and environmentally friendly. Thus, PMMA can be used in making economical, phase change microcapsules. In the continuous exploration of phase change microcapsules by many scholars, the phase change microcapsules with PMMA as the shell material and paraffin as the core material have been further developed in terms of the synthesis method, the ratio of core material to shell material, and the characterization method (Alay et al. 2011; Alkan et al. 2011; Han et al. 2015; Xu and Yang 2019).

Microencapsulated phase change materials are used in a wide range of fields. In the field of architectural coatings, they are often used in cement mortar, concrete, walls, floors, and other building materials to achieve heat storage, cooling, energy saving, and other functions (Feldman et al. 1991; Pasupathy et al. 2008; Hunger et al. 2009; Jeong et al. 2012). Yu and Pei (2020) prepared coatings with thermoregulation function by using methyl methacrylate and ethyl methacrylate as wall raw materials and paraffin wax encapsulation by suspension polymerization. In furniture coatings, research related to microencapsulation technology has gradually increased. Yan et al. (2019a,b, 2021a,b) have conducted considerable research on the effect of microcapsules added to coatings on the properties of paint films, which makes the study on the characterization of the properties of microcapsules added to wood furniture coatings more and more mature. At present, there are more studies that have been carried out on the mechanism of phase change microcapsules and coating preparation, but there has been a lack of published work on the applications of phase change microcapsule coatings to wooden furniture surfaces, and the use of phase change properties of phase change microcapsules to achieve the cooling effect.

In this study, after mixing PCM powder with water-based varnish for wood furniture in certain proportions, the effect of adding different proportions of PCM on the thermal properties of the coating was analyzed. Next, after the prepared coatings were applied to the MDF surface, the effect of PCM addition on the hardness, adhesion, and 
gloss of the water-based paint film was tested. Finally, through use of a homemade device to simulate the constant temperature of the human body exothermic process, further exploration of the heat absorption and cooling function of the paint is needed to understand whether it can be sent to the requirements of the regulation of temperature awareness.

\section{EXPERIMENTAL}

\section{Materials}

The wood furniture material used in this experiment was medium density fiberboard (MDF) with a density of $778 \mathrm{~kg} / \mathrm{m}^{3}$ and dimensions of $500 \times 500 \times 20 \mathrm{~mm}$. The core material of the phase change microcapsules was paraffin, and the shell material was polymethyl methacrylate. The water-based varnish for surface finishing was a modified polyvinyl acetate-ethylene copolymer water-based varnish. The main experimental apparatus is shown in Table 1.

Table 1. Main Test Instruments

\begin{tabular}{|c|c|c|c|c|}
\hline $\begin{array}{c}\text { Instrument Name } \\
\begin{array}{c}\text { Scanning electron } \\
\text { microscope }\end{array}\end{array}$ & $\begin{array}{c}\text { FEI Quanta } \\
200\end{array}$ & Manufacturers & City & Country \\
\hline $\begin{array}{c}\text { Differential scanning } \\
\text { calorimeter }\end{array}$ & DSC 214 & $\begin{array}{c}\text { NETZSCH-Gerätebau } \\
\text { GmbH }\end{array}$ & Shanghai & China \\
\hline Adhesion tester & $\begin{array}{c}\text { QFH- } \\
\text { HG600 }\end{array}$ & $\begin{array}{c}\text { Dongguan Huaguo } \\
\text { Precision Instrument Co. }\end{array}$ & Dongguan & China \\
\hline $\begin{array}{c}\text { Combined pencil } \\
\text { hardness tester }\end{array}$ & $\begin{array}{c}\text { QHQ/B- } \\
3084\end{array}$ & $\begin{array}{c}\text { Dongguan Huaguo } \\
\text { Precision Instrument Co. }\end{array}$ & Dongguan & China \\
\hline $\begin{array}{c}\text { Three-angle } \\
\text { glossmeter }\end{array}$ & HG268 & $\begin{array}{c}\text { Shenzhen ThreeNH } \\
\text { Technology Co. }\end{array}$ & Shenzhen & China \\
\hline $\begin{array}{c}\text { Multi-channel } \\
\text { temperature logger }\end{array}$ & PT700-16 & $\begin{array}{c}\text { Shenzhen Toprie } \\
\text { Electronics Co. }\end{array}$ & Shenzhen & China \\
\hline
\end{tabular}

The phase change microencapsulated coating cooling performance test apparatus was a homemade apparatus, simulating the human forearm exothermic device, and its external dimensions were $200 \times 30 \times 20 \mathrm{~mm}$. It consisted of five parts, as shown in Fig. 1: (1) digital display thermostatic water bath (HH-2); (2) adjustable speed peristaltic pump; (3) latex tube; (4) simulated human upper limb quartz square bottle; and (5) simulated human skin of $2 \mathrm{~mm}$ elastic silicone rubber plate. The device worked by circulating the temperature of water to simulate the temperature of the upper limb to achieve the effect of constant temperature and exotherm.

\section{Methods}

Human temperature sensory test contact experiment

Ten adult males and females, aged between 20 and 30 years old, were selected as test subjects who felt no significant abnormalities in temperature and humidity. The experiments were conducted using the palm of the upper limb in contact with the experimental material (Fig. 2), and the temperature acquisition test points were selected from the tip of the middle finger $\left(T_{1}\right)$, the greater interphalangeal muscle of the palm $\left(T_{2}\right)$, and the lateral side of the forearm ( $\left.T_{3}\right)$, respectively (Fig. 3) (Wang et al. 2000). This worked by connecting the temperature sensor on the multiplex temperature tester at the test 
point and by testing the contact temperature at each of the three points. The laboratory temperature was set at $27{ }^{\circ} \mathrm{C}$ and the humidity was $50 \% \pm 10$. After the test started, the subject's upper limbs were in contact with the test material in a natural fit, and the temperature change at three points was recorded for $30 \mathrm{~min}$. The average taken of the three test points temperature was the test temperature $T$.

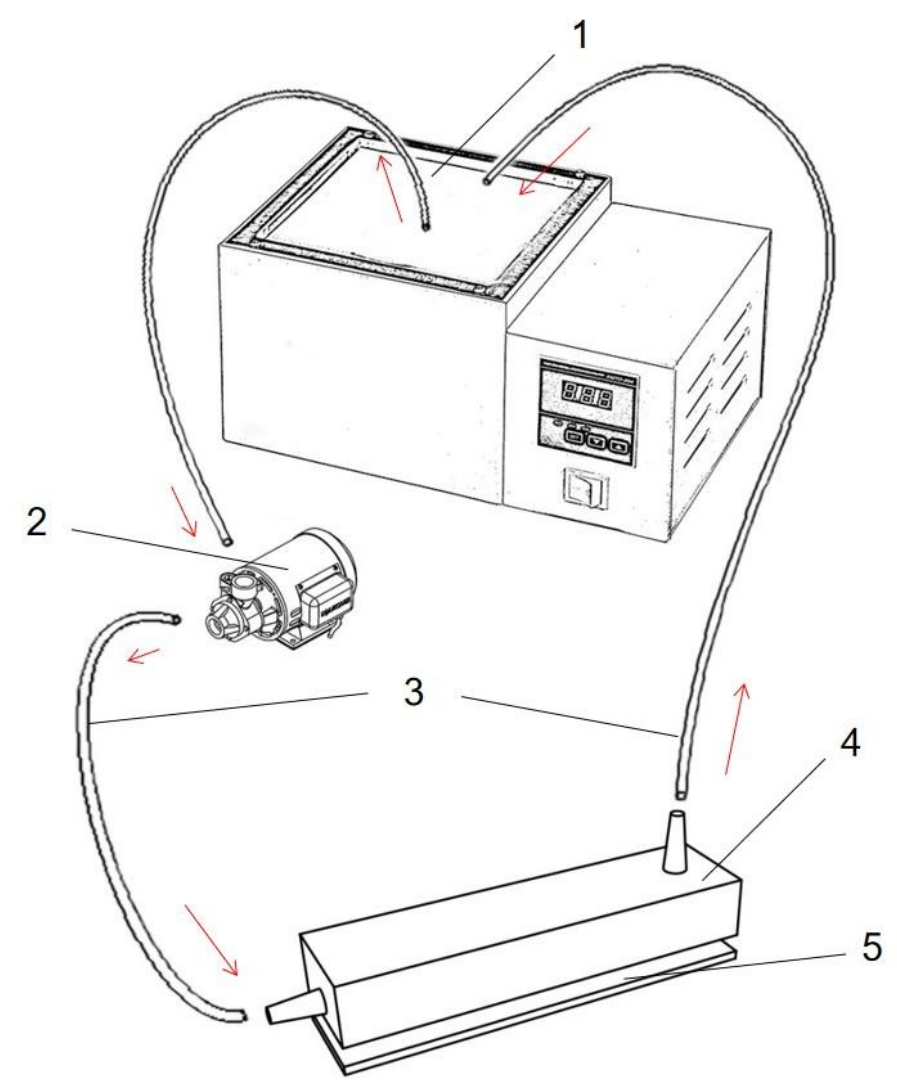

Fig. 1. Parts of cooling performance testing devices

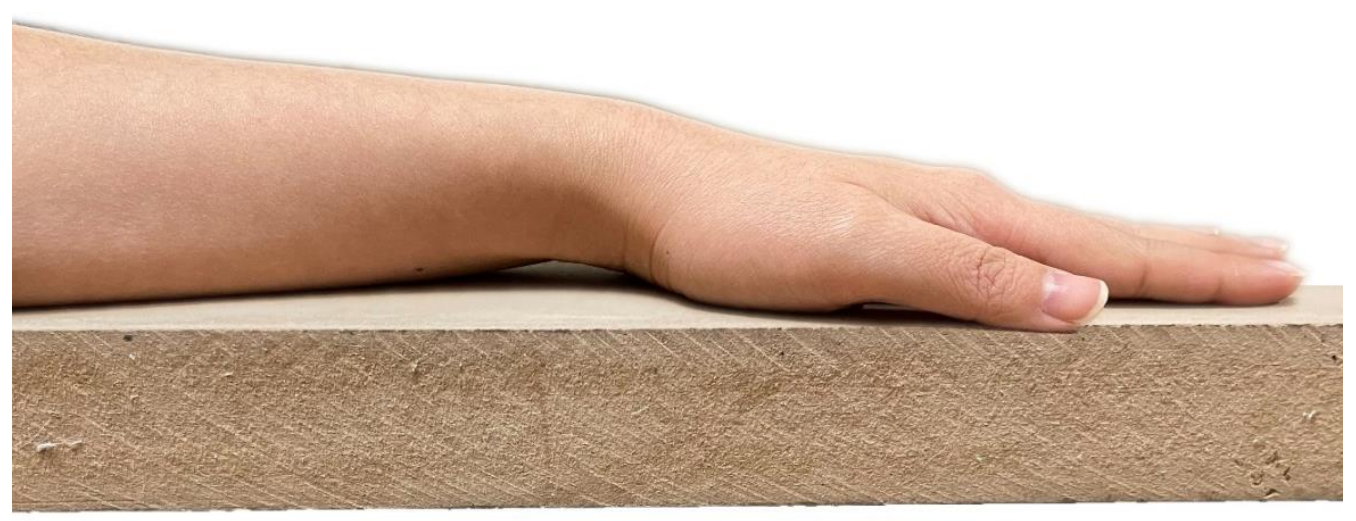

Fig. 2. Hand position on the furniture surface 


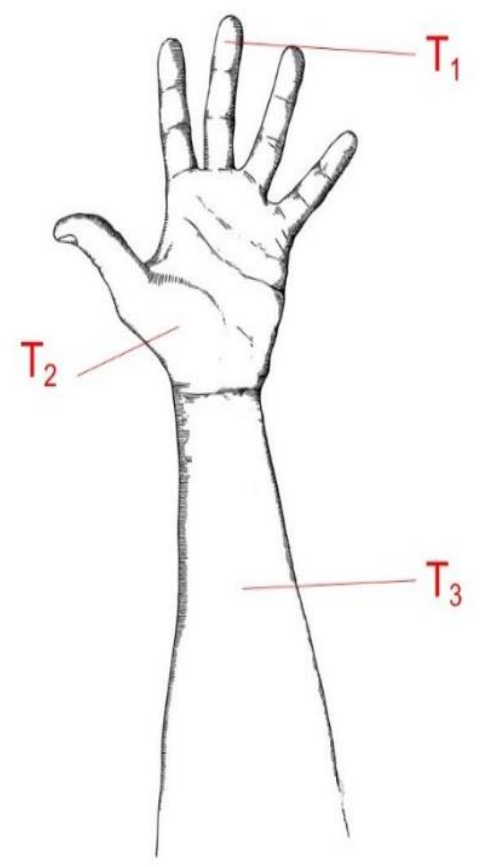

Fig. 3. Temperature test points on the hand of the subjects

The subjective method was used to test the thermo-perceptual properties of the wooden tabletop while testing the temperature. The subjects rated the predicted mean vote $(P M V)$ of the material on a scale of "cold (-3 points), cool (-2 points), slightly cool (1 point), comfortable (0 points), slightly hot (1 point), warm (2 points), and hot (3 points)" at one-minute intervals (Lan et al. 2019).

Preparation of phase change microencapsulated coatings and lacquer films

In this test, PCM was mixed with water-based varnish in the proportions of 5\%, $10 \%, 15 \%$, and $20 \%$ by mass, and the appropriate amount of dispersant was added and mixed well with high-speed stirring. After stirring well, a brush was used to apply the appropriate amount of varnish and spread it evenly on the surface of MDF board with a finishing amount of $150 \mathrm{~g} / \mathrm{m}^{2}$. According to the wood furniture finishing requirements, after finishing three layers (one primer and two topcoats), the wood furniture was placed at room temperature environment to cure and dry for more than 7 days. After the paint was completely dry, its performance was tested.

\section{Phase change microcapsules and paint film characterization for coatings}

The particle size and morphological characteristics of the microcapsules were analyzed by scanning electron microscopy (SEM), followed by the analysis of the surface characteristics of the paint film after film formation of the waterborne paint.

The thermal properties can be determined by recording the heat absorbed or released by the microcapsules and varnish film as a function of time using a differential scanning thermal analyzer (DSC) to determine the phase change capability of the phase change microcapsules. The temperature range was -30 to $180{ }^{\circ} \mathrm{C}$ with nitrogen as the protective gas and the flow rate of the protective gas was $40 \mathrm{~mL} / \mathrm{min}$ and the flow rate of the purge gas was $60 \mathrm{~mL} / \mathrm{min}$. 
Phase change microencapsulated waterborne coating film performance test

To observe the effect of the addition of PCM powder on the paint film adhesion, the method of the paint film adhesion test was carried out according to ISO 2409 (2020). A hand-held multi-blade cutting tool was used to characterize the degree of peeling of the paint film after cross-cutting.

The gloss of the paint film was tested using a three-angle gloss meter with reference to ISO 2813 (2014). The gloss reflectance of the paint film was characterized by the gloss reflectance at different angles. Referring to ISO 15184 (2012), to observe the effect of the PCM powder addition on the hardness of paint films, the hardness was characterized by pencil type.

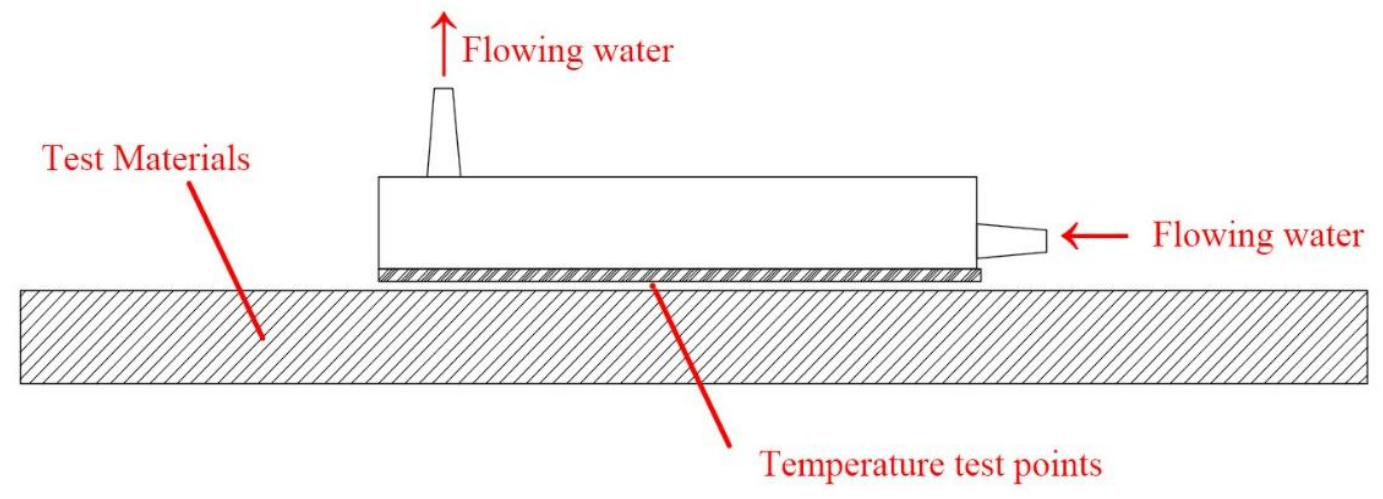

Fig. 4. Schematic diagram of the simulated temperature test of the device

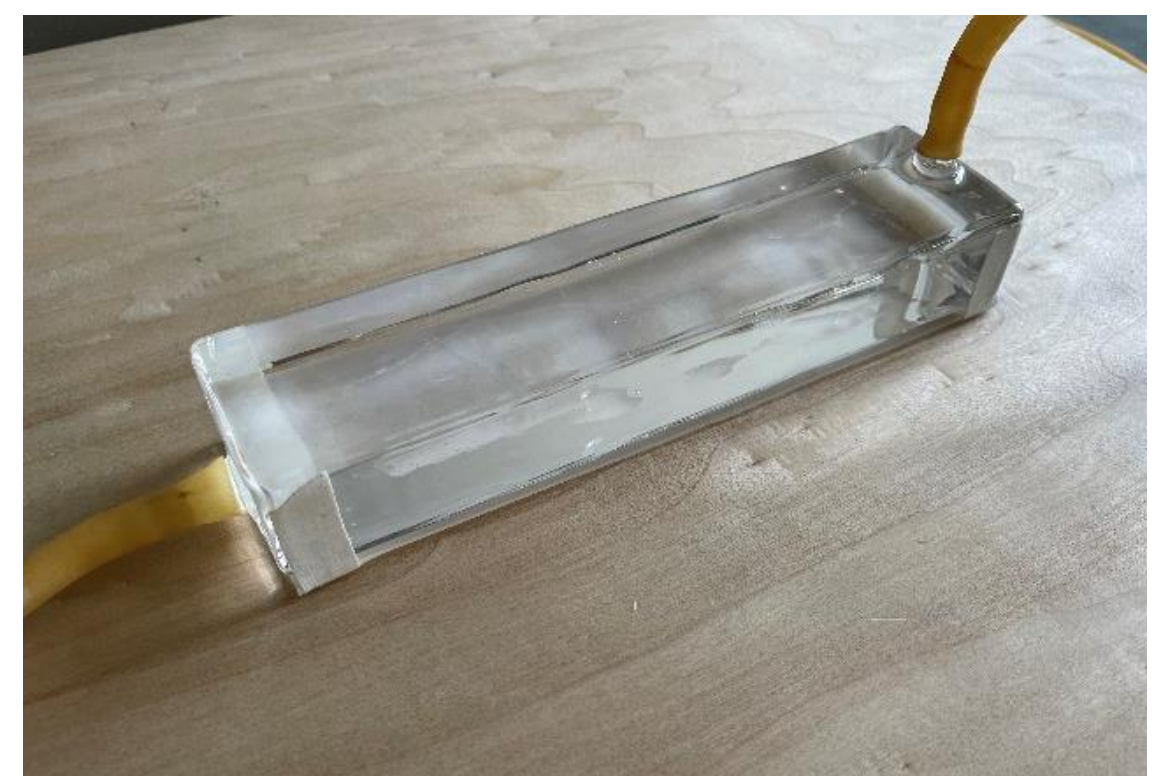

Fig. 5. Quartz square vial for microcapsules cooling performance test

Phase change microcapsules cooling performance test

The cooling performance of PCM coatings was carried out using homemade equipment. The temperature of the constant temperature water bath was set to $39.5^{\circ} \mathrm{C}$ with temperature fluctuations $\leq 0.5^{\circ} \mathrm{C}$. After the temperature of the bath reached $39.5^{\circ} \mathrm{C}$, the peristaltic pump with adjustable speed was turned on, and the volume flow rate was set to $2.52 \mathrm{~m}^{3} / \mathrm{h}$. Water flowed in through the bottom inlet of the quartz square vial and out through the top outlet above, and the temperature of the silicone rubber surface increased 
and finally stabilized (Fig. 4). The physical quartz square vial is shown in Fig. 5. The temperature receiver of the multiplex temperature tester was connected to the silicone rubber surface in contact with the painted MDF and the temperature change was recorded over a period of $25 \mathrm{~min}$. The ambient temperature of the laboratory room was $27 \pm 0.5^{\circ} \mathrm{C}$, and the evaluation rate of the multiplex temperature tester test was once every $6 \mathrm{~s}$.

\section{RESULTS AND DISCUSSION}

\section{The Best Human Comfort Temperature Range}

The test temperature $T$ obtained in the human temperature sensory test experiment and the temperature sensory evaluation $P M V$ can be a function of time. In other words, each minute of contact temperature $T$ corresponds to a value of $P M V$, and there is a certain relationship between $T$ and $P M V$. A linear regression was performed in SPSS (General Linear Model, SPSS Inc., Chicago, IL, USA) with $P M V$ as the dependent variable and temperature $T$ as the independent variable. The coefficient of determination after regression, $\mathrm{R}^{2}=0.797$, was initially judged to be a good linear fit. The significance of ANOVA, $\mathrm{P}=0<0.01<0.05$, indicated that the linear relationship regression model established by the independent variable $T$ and the dependent variable $P M V$ was highly statistically significant, i.e., the linear relationship was significant. Then, the linear regression equation was established as Eq. 1.

$$
P M V=1.015 T-33.99
$$

where $P M V$ is the predicted mean vote and $T$ is the human contact temperature $\left({ }^{\circ} \mathrm{C}\right)$. The optimal threshold value of $P M V$ was between -0.5 and 0.5 , and it started to feel uncomfortable when it exceeded \pm 1 . By Eq. 1, the authors found the contact temperature $T$ corresponding to \pm 1 . When the human contact temperature reached about $32.5^{\circ} \mathrm{C}$, the $P M V$ reached -1 . When the human contact temperature reached about $34.5{ }^{\circ} \mathrm{C}, P M V$ reached 1 . When the human contact temperature reached about $33.5^{\circ} \mathrm{C}$, the $P M V$ reached 0 . Therefore, the cooling paint should try to make the human contact temperature close to $33.5^{\circ} \mathrm{C}$ and not more than $34.5^{\circ} \mathrm{C}$.

\section{Analysis of the Structure and Thermal Properties of Phase Change Microcapsules}

To further understand the surface morphology of the phase change microcapsule powder, the PCM samples were analyzed by scanning electron microscopy. Figures 6 and 7 show the SEM test results at $250 \times$ and $3000 \times$ field of view, respectively. As can be seen from the figure, the powder microcapsules had a clustered morphological appearance with smooth surface and mostly irregular spherical structure, and the average particle size was $25 \mu \mathrm{m}$. Since at $32.5^{\circ} \mathrm{C}$, the thermal comfort evaluation of the human body reaches the minimum comfort threshold, which is the lower limit of comfortable temperature, microcapsules with phase change at around $32{ }^{\circ} \mathrm{C}$ were selected as the additive material. The heat flow-temperature curve was obtained by DSC test, as shown in Fig. 8. The microcapsules reached the phase transition peak at $32.22^{\circ} \mathrm{C}$, indicating that the solid-liquid phase transition occurred during heat absorption. The enthalpy of phase change is the integral of the area enclosed by the curve and the auxiliary line divided by the heating rate, which was calculated to be $209.0 \mathrm{~J} / \mathrm{g}$. The PCM powder had a large enthalpy of phase change and could absorb more heat. 


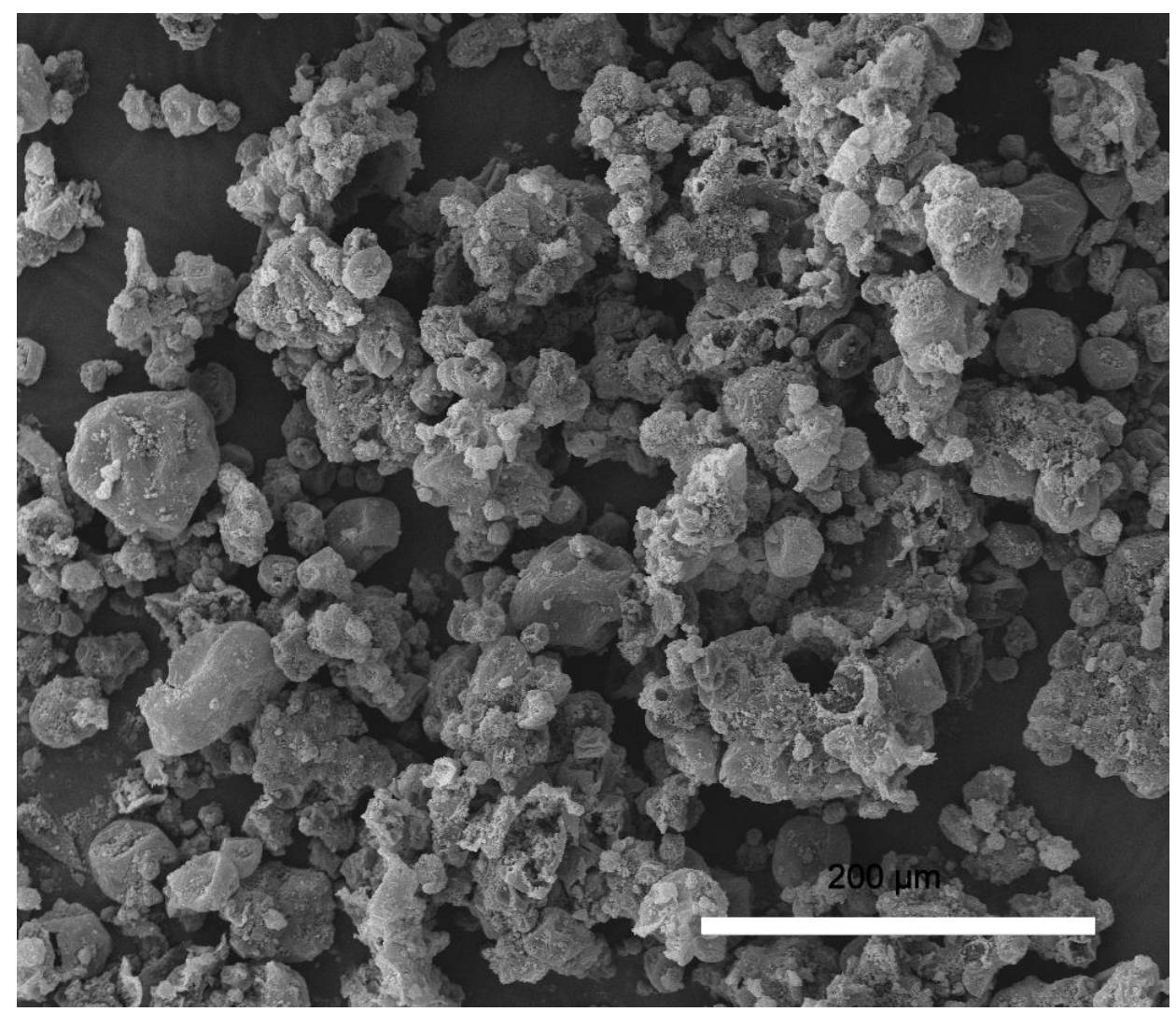

Fig. 6. $250 \times$ phase change microcapsule electron microscopy

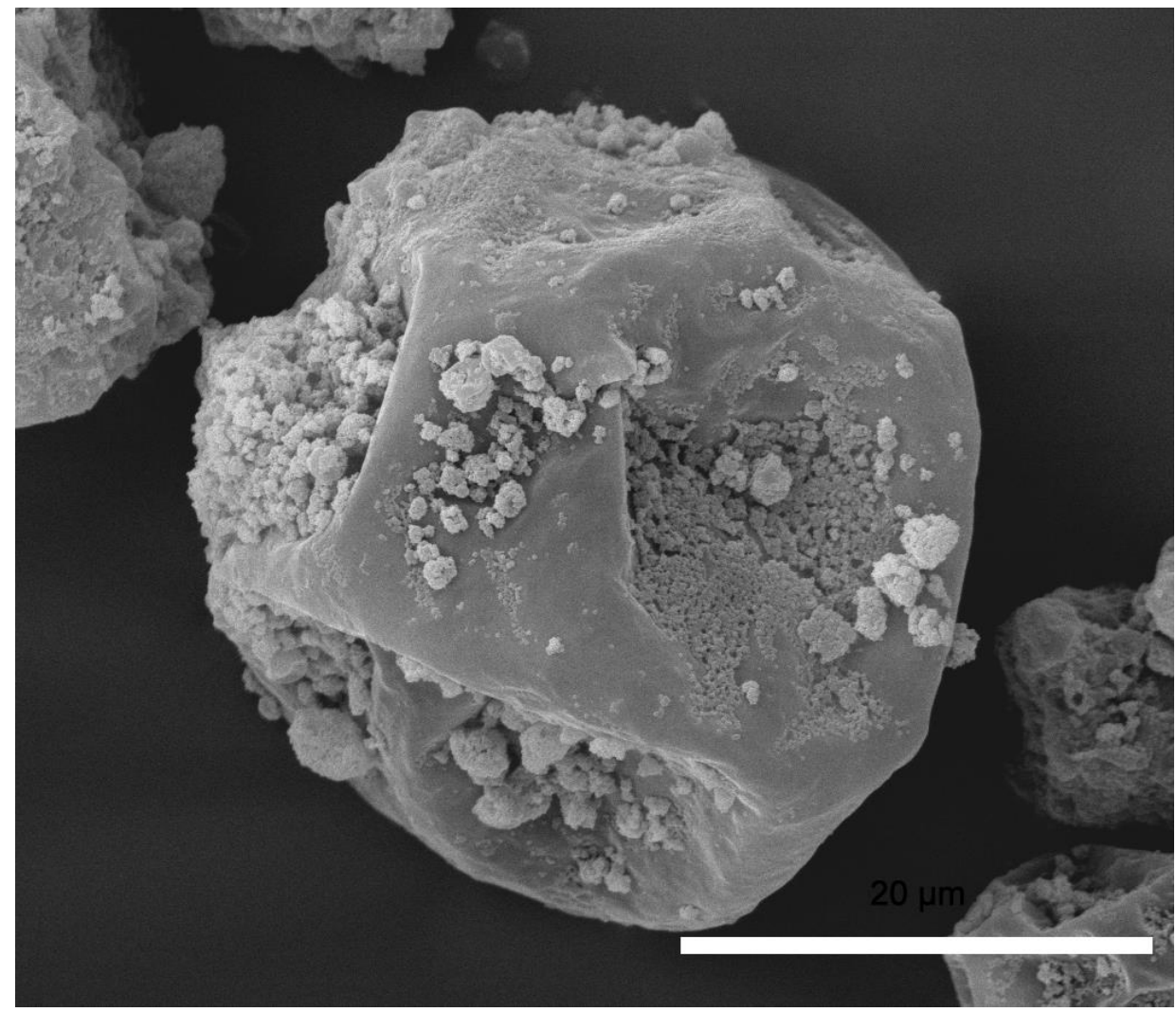

Fig. 7. $3000 \times$ phase change microcapsule electron microscopy 


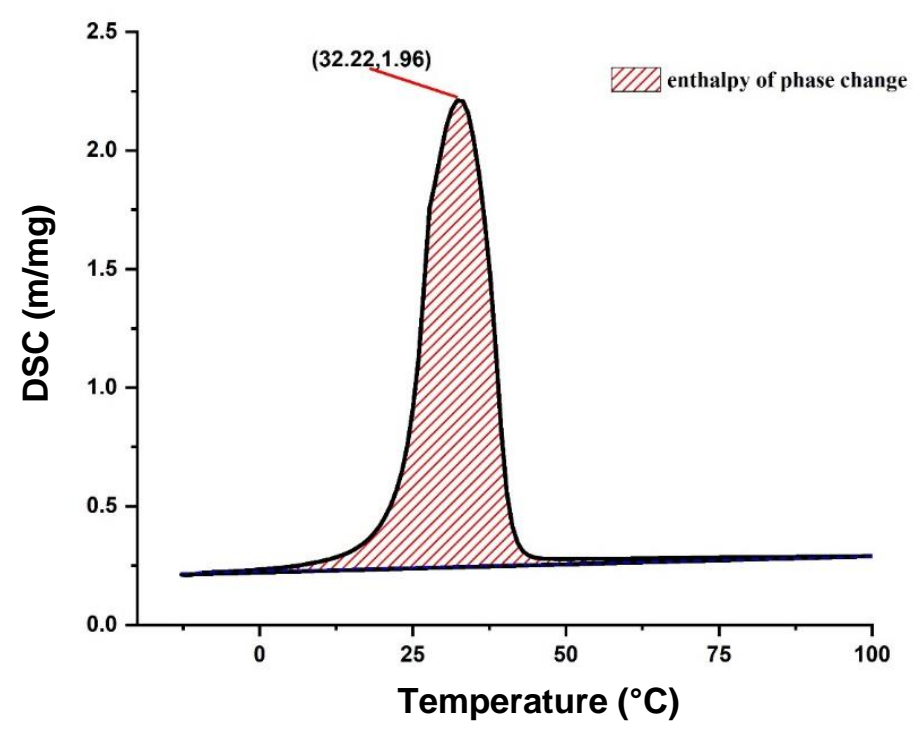

Fig. 8. Diagram of differential scanning calorimetry analysis of phase change microcapsules

\section{Analysis of Paint Film Structure and Thermal Properties}

Scanning electron microscopy analysis of the paint film surface of PCM samples with different solid contents is shown in Fig. 9. From the figure, it can be seen that the surface of the varnish film without adding PCM powder was relatively flat except for a small amount of impurities. As the amount of powder added increased, the surface of the paint film became rough and some of the powder appeared agglomerated. In general, PCM powder was more uniformly dispersed in the varnish, with relatively little agglomeration and a more stable film structure.

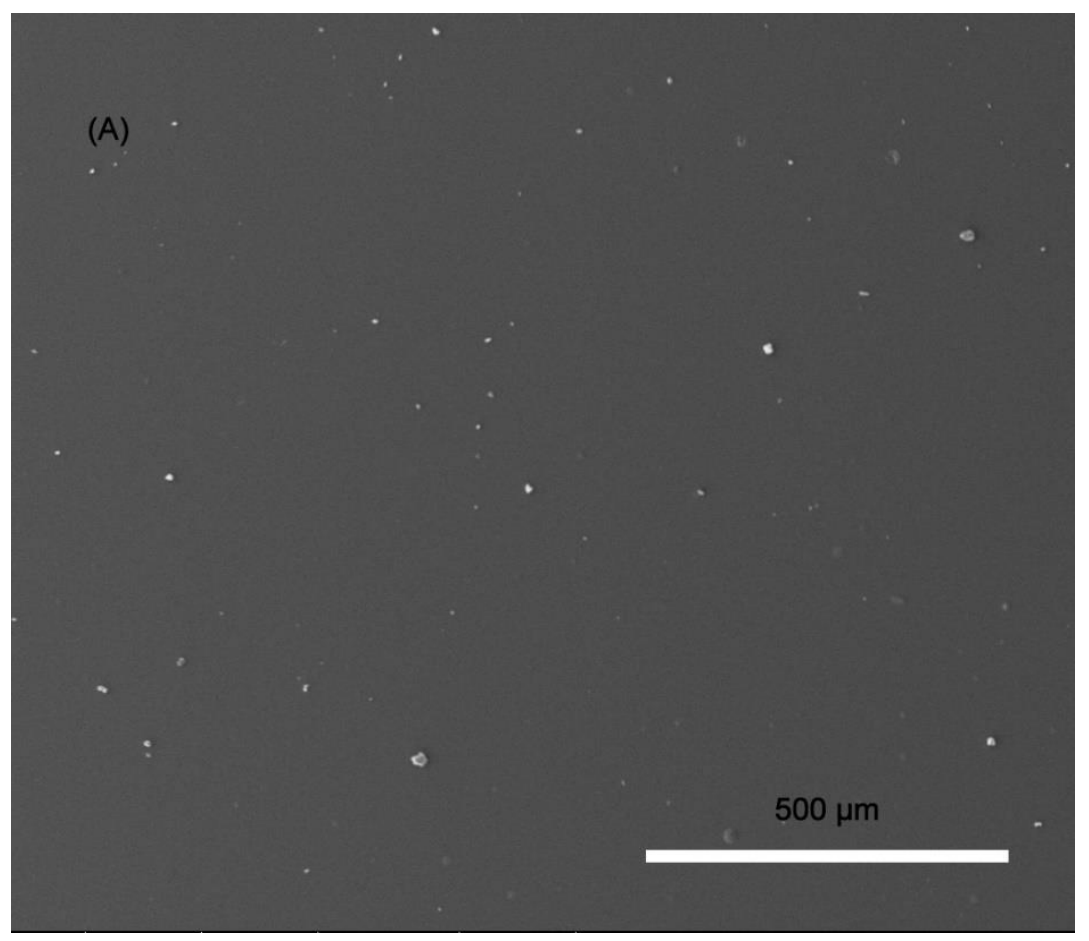



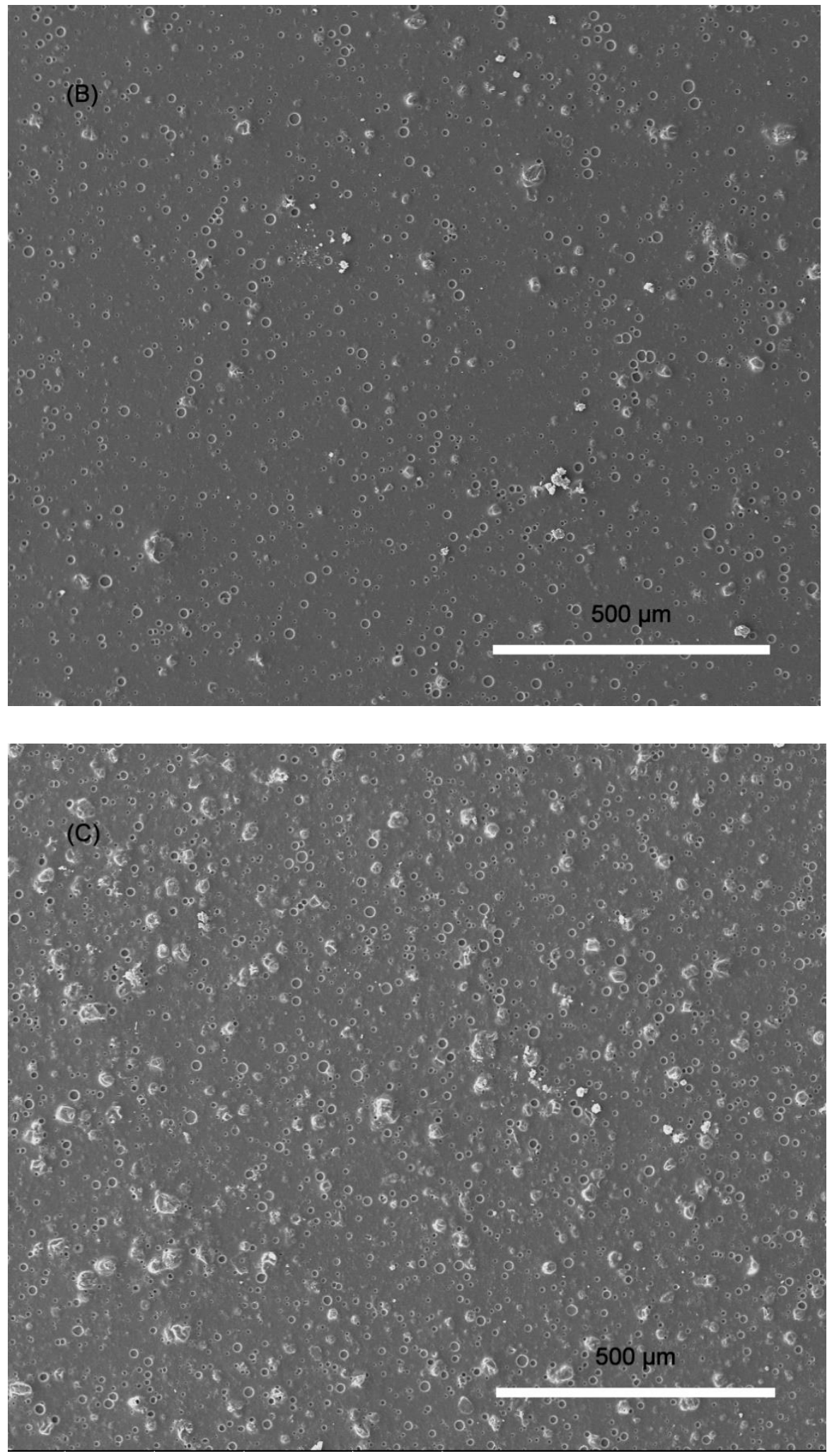

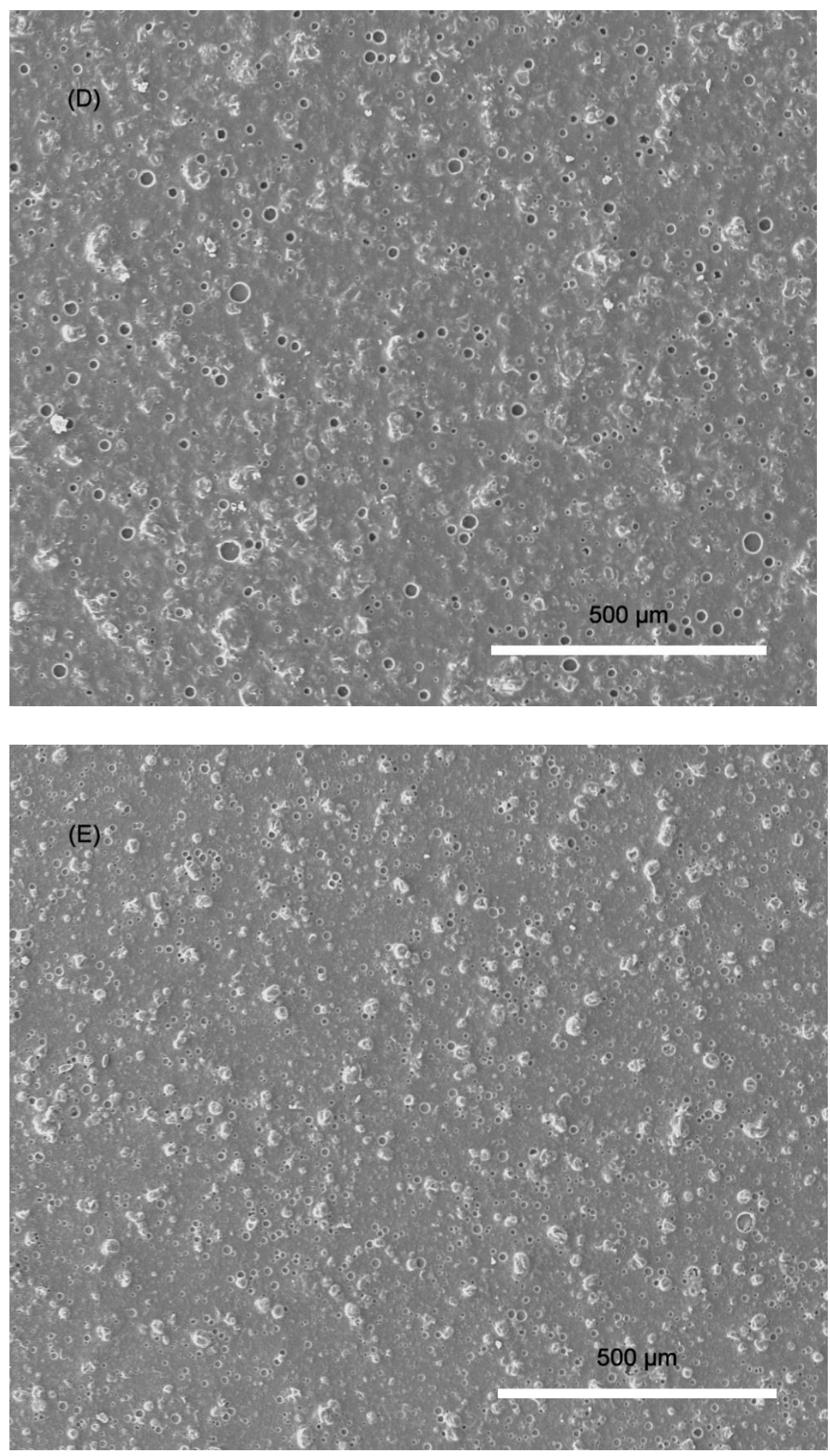

Fig. 9. Microstructure of different paint film surfaces under scanning electron microscopy; A. Waterbased varnish film; B. 5\% solid content PCM water-based paint film; C. 10\% solid content PCM water-based paint film; D. 15\% solid content PCM water-based paint film; E. $20 \%$ solid content PCM water-based paint film 
The paint films with different contents were analyzed by DSC, as shown in Fig. 10. It can be seen from the figure that the DSC of the paint film without the addition of PCM powder increased with the increase of temperature and did not show any peak, and there was no phase change. All of the paint films added with PCM powder showed phase change peaks, and all of them exhibited phase change heat absorption. With the increase of PCM powder addition, the peak phase transition increased continuously and the enthalpy of phase transition also increased. That is, as the solid content increased, the phase change capacity of the paint film increased and the amount of heat that could be absorbed increased.

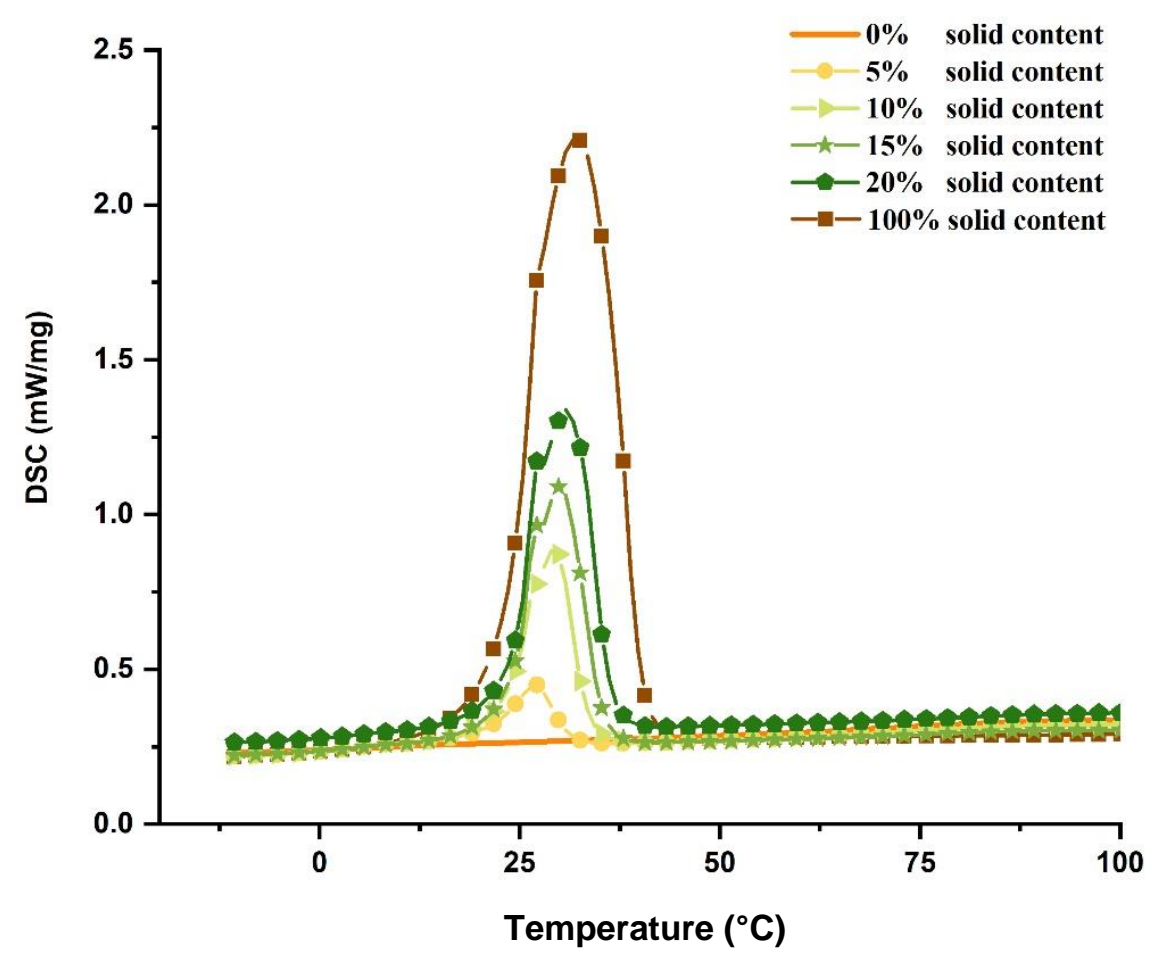

Fig. 10. Heat flow-temperature (DSC) curves for different paint films

\section{Paint Film Performance Analysis}

The paint film adhesion grade of PCM powder and varnish mixed with the finishing material is shown in Table. 2. The PCM powder did not cause a negative effect on the paint film adhesion, and it even enhanced the paint film adhesion with the increase of the microcapsules content.

Table 2. Paint Film Adhesion Grade

\begin{tabular}{|c|c|c|c|c|c|}
\hline PCM Content & $0 \%$ & $5 \%$ & $10 \%$ & $15 \%$ & $20 \%$ \\
\hline Grade & 2 & 2 & 2 & 1 & 1 \\
\hline
\end{tabular}

The gloss of the paint film after PCM powder was added to water-based paint is shown in Table. 3. From the table, it can be seen that the film of water-based paint before adding powder was medium gloss, while the gloss of the film dropped rapidly after adding powder. According to ISO 2813 (2014), the gloss level below 10 in the case of the $60^{\circ}$ 
angle test was low gloss film, and the data at the observation angle of $85^{\circ}$ was more accurate. The gloss of the paint film decreased gradually with the increase of PCM content in the case of the $85^{\circ}$ observation incidence angle, and the gloss was relatively close after $10 \%$ content.

Table 3. Glossiness of Paint Film

\begin{tabular}{|c|c|c|c|}
\hline \multirow{2}{*}{ PCM Content } & \multicolumn{3}{|c|}{ Glossmeter Incidence Angle Degree } \\
\cline { 2 - 4 } & $\mathbf{2 0}^{\circ}$ & $\mathbf{6 0}^{\circ}$ & $\mathbf{8 5}^{\circ}$ \\
\hline $0 \%$ & 15.3 & 44.9 & 54.6 \\
\hline $5 \%$ & 2 & 9.4 & 8.2 \\
\hline $10 \%$ & 0.8 & 3.3 & 2 \\
\hline $15 \%$ & 0.9 & 2.9 & 1.6 \\
\hline $20 \%$ & 1 & 2.5 & 1.4 \\
\hline
\end{tabular}

As the content of phase change microcapsules increased, the hardness of the paint film decreased (Table. 4). By $20 \%$ of the content, the hardness decreased to $2 \mathrm{~B}$, which was already lower than the standard hardness of the paint film.

Table 4. Paint Film Hardness

\begin{tabular}{|c|c|c|c|c|c|}
\hline PCM Content & $0 \%$ & $5 \%$ & $10 \%$ & $15 \%$ & $20 \%$ \\
\hline Hardness & $2 \mathrm{H}$ & $2 \mathrm{H}$ & $\mathrm{H}$ & $\mathrm{B}$ & $2 \mathrm{~B}$ \\
\hline
\end{tabular}

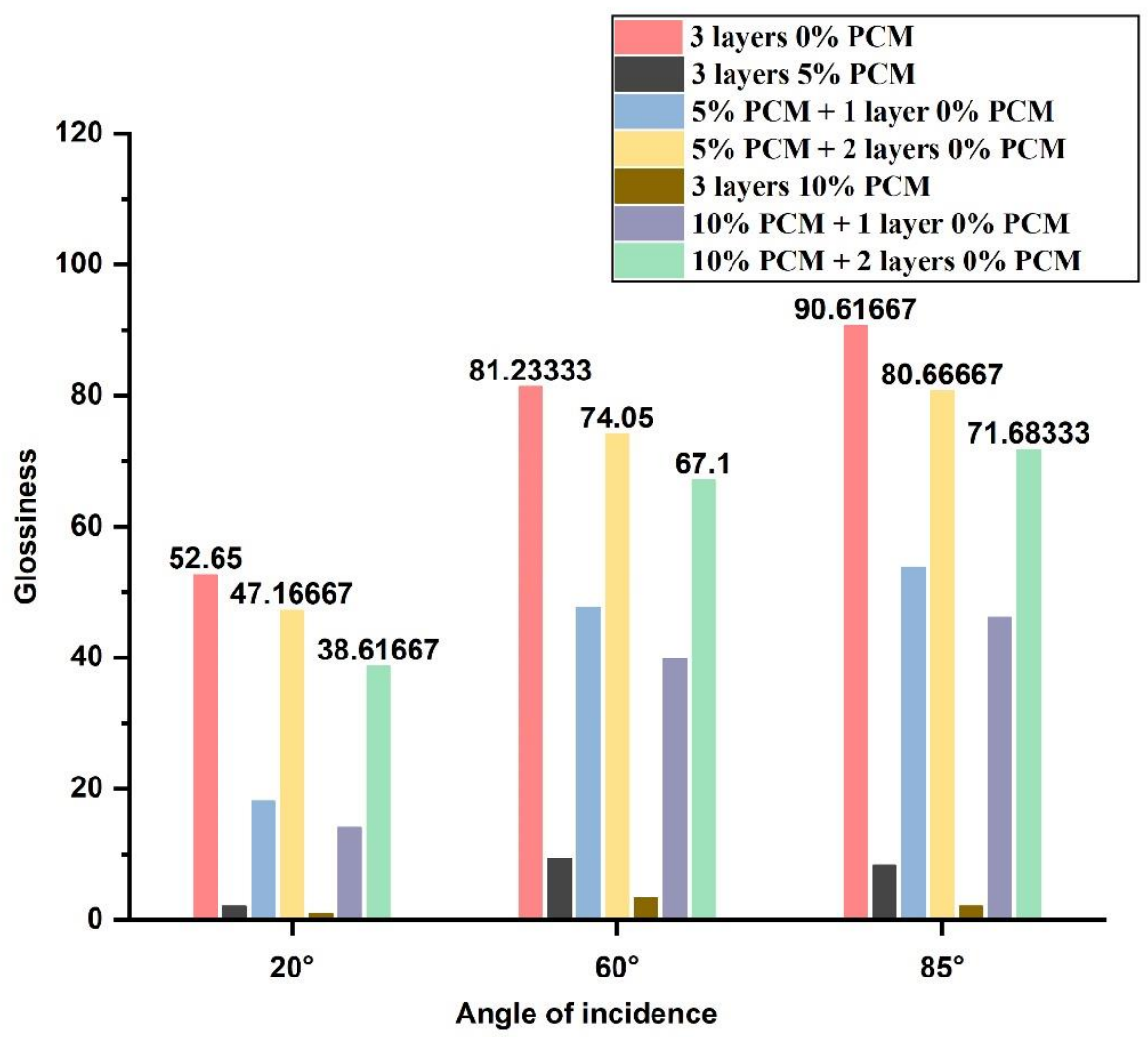

Fig. 11. Comparison of glossiness after adding PCM in layers 
In terms of paint film performance, the hardness of the $15 \%$ and $20 \%$ solid content was too low to meet the finishing requirements of wood furniture. And the PCM had a great influence on the gloss after finishing, so the two solid contents of $5 \%$ and $10 \%$ were not suitable for the actual application of the paint film. To improve the gloss, PCM was added to the primer only, and no PCM was added to the topcoat, as shown in Fig. 11. After the superimposed topcoat, it can obviously alleviate the effect of PCM powder addition on gloss, and the gloss increased. The gloss level after adding two topcoats was close to that of the film without PCM powder.

\section{Phase change microencapsulated paint film temperature reduction performance}

A home-made device was used to simulate the cooling performance of coatings of phase change microencapsulated materials. As can be seen from Fig. 12, when PCM powder was not added, the contact temperature of the painted MDF rose faster after heating at $27{ }^{\circ} \mathrm{C}$ room temperature. The contact temperature reached $32.51^{\circ} \mathrm{C}$ at $1.1 \mathrm{~min}$ and 34.51 ${ }^{\circ} \mathrm{C}$ at $12.1 \mathrm{~min}$. Therefore, only between 1.1 and $12.1 \mathrm{~min}$ within $30 \mathrm{~min}$ belonged to a more comfortable temperature range. In the higher indoor temperature conditions, wood furniture materials after ordinary coating could only maintain the cooling effect for a short period of time. After a little longer contact time, the temperature sensation was no longer comfortable, and it even produced a stifling feeling.

After the addition of PCM powder, the contact temperature dropped significantly, and the cooling effect was obvious. All coatings to which PCM powder was added did not exceed $34.5{ }^{\circ} \mathrm{C}$ within $30 \mathrm{~min}$, and always remained below the comfort limit. Therefore, the addition of PCM powder not only made the overall contact temperature drop, and in the long-term contact, it continuously absorbed heat to achieve the cooling effect.

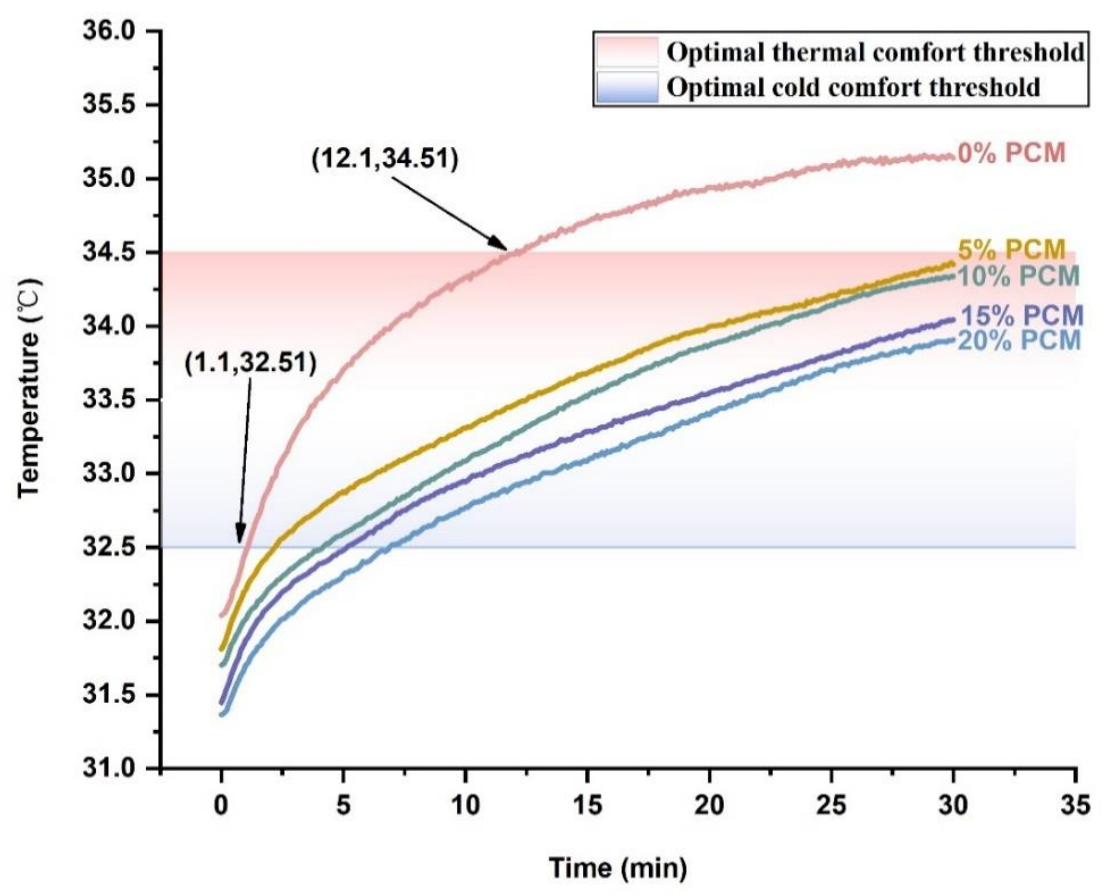

Fig. 12. Temperature-time curve of simulated heat transfer device 
Since the hardness of the paint film was too low to meet the furniture coating requirements at $15 \%$ and $20 \%$ solid content, and the cooling effect could already be achieved at $5 \%$ addition, the cooling of the paint at $5 \%$ and $10 \%$ solid content was further studied. A certain amount of PCM was added to the primer, and the topcoat without PCM was brushed on after the primer was solidly dried, and the temperature reduction was tested as shown in Figs. 13 and 14.

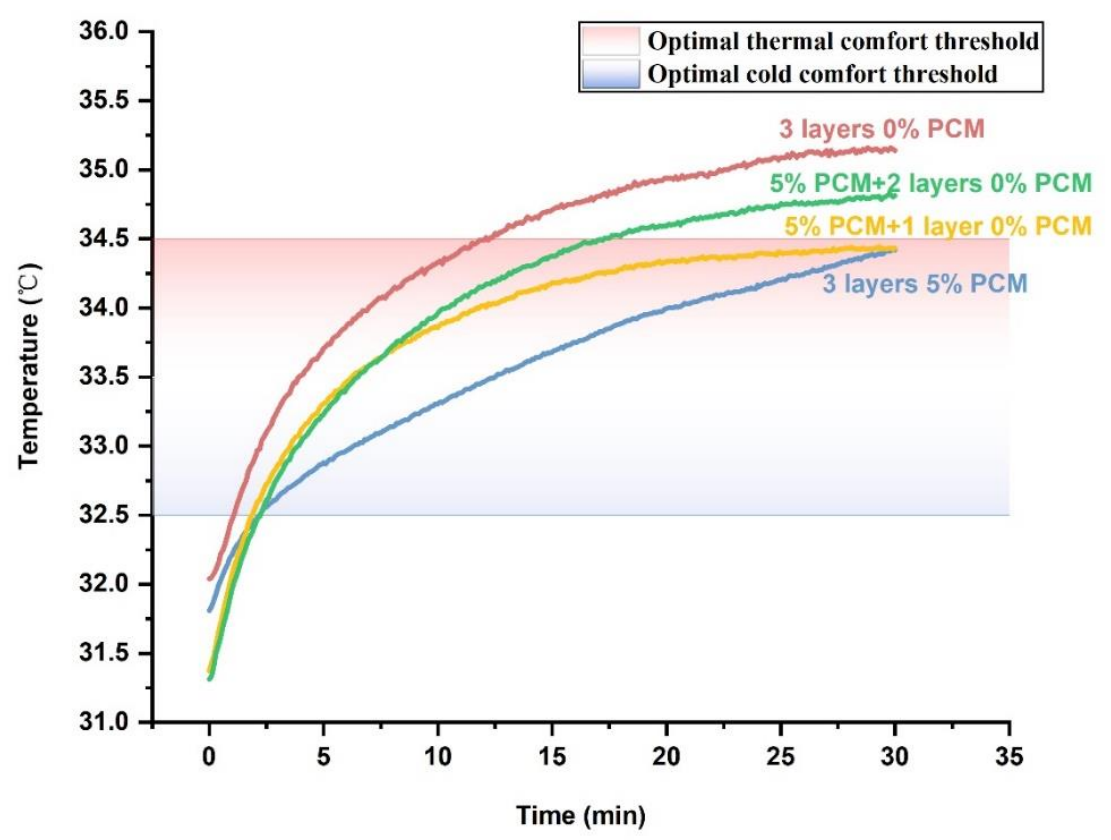

Fig. 13. Comparison chart of contact temperature change during $5 \%$ solids PCM layered finishing

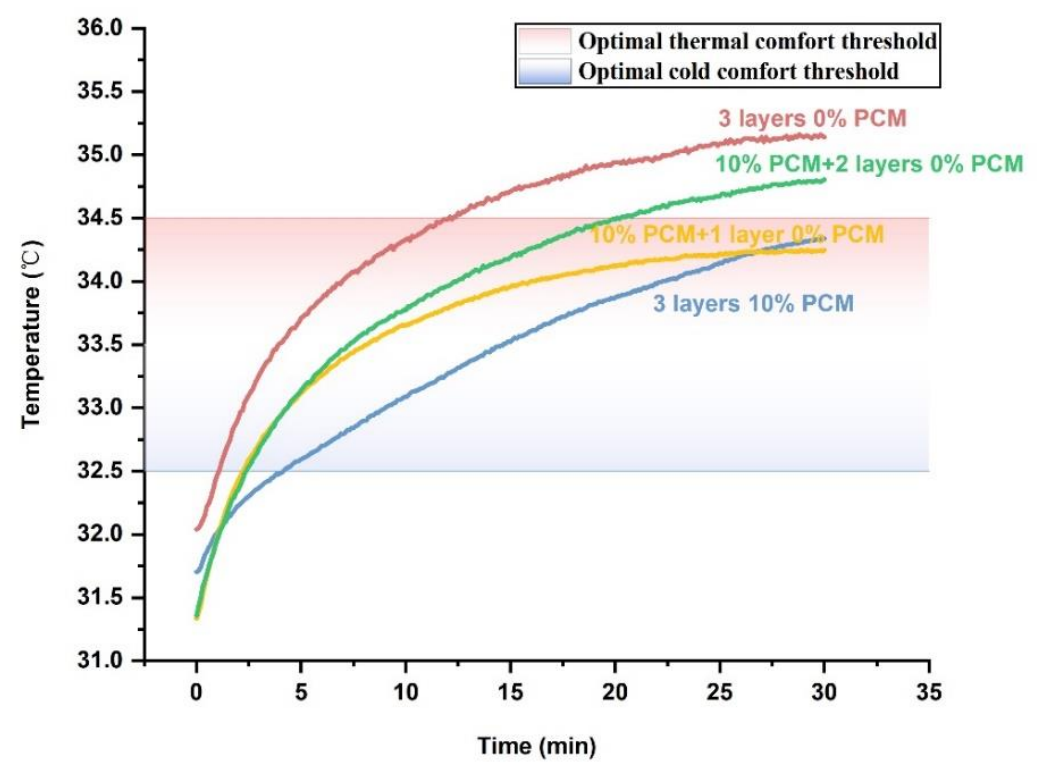

Fig. 14. Comparison chart of contact temperature change during $10 \%$ solids PCM layered finishing 
The contact temperatures of the two solid contents showed the same pattern. In other words, even after overlaying the topcoat, the PCM in the primer could still play a cooling role, but with the overlay of the topcoat, the cooling effect was reduced. In actual production, the number of layers of topcoat can be selected according to different process requirements.

\section{CONCLUSIONS}

1. Microscopically, phase change microcapsule (PCM) powder with paraffin as the core material and PMMA as the shell material was mostly irregularly spherical in appearance, with good inclusions and an average particle size of $25 \mu \mathrm{m}$. Thermal analysis by DSC showed that the peak phase transition of PCM powder was at $32.2^{\circ} \mathrm{C}$ and the enthalpy of phase transition was $209.0 \mathrm{~J} / \mathrm{g}$, which is a good effect of phase transition heat absorption.

2. After adding PCM powder into the water-based varnish, the dispersion in the varnish was more uniform, but it made the surface of the paint film rough. With the increase of PCM addition, some microcapsules appeared to be agglomerated. From a macroscopic point of view, the mixing of water-based varnish with phase change microencapsulated powder did not cause any adverse effect on the adhesion of the paint film. However, PCM had a great influence on the gloss of the paint film, and a small amount of added gloss dropped sharply, and the matting phenomenon was obvious. As the content of PCM powder increased, the hardness of the paint film decreased continuously to $2 \mathrm{~B}$ at $20 \%$ content, which already did not meet the basic requirements of the paint film. Referring to the wood furniture finishing method, adding PCM powder to the primer only, and then overlaying the topcoat without PCM significantly mitigated the problem of decreasing gloss of the paint film.

3. The phase change ability was also obtained for the waterborne varnish with the addition of PCM powder. After DSC analysis, it was found that the enthalpy of phase change increased with the increase of powder addition, and the heat that could be absorbed also increased. Using a homemade device, the cooling performance of the paint was simulated at $27^{\circ} \mathrm{C}$ room temperature for $30 \mathrm{~min}$, and it was found that the contact temperature of the paint film without PCM powder exceeded the upper limit of the comfort temperature at $12.1 \mathrm{~min}$ and started to produce an uncomfortable temperature sensation experience. After adding PCM powder, the contact temperature decreased significantly, and the higher the powder solid content, the lower the contact temperature. The contact temperature of the added PCM powder did not exceed the upper limit of the comfortable temperature, which means that even a small amount of addition can achieve a good cooling effect. Further research was conducted on the case of adding PCM to the primer only. The experiments demonstrated that adding PCM powder to the primer only and then overlaying the topcoat can still achieve the cooling effect, but the increase in the number of overlaying layers made the cooling effect decrease.

4. Taken together, PCM powder added to water-based varnish for wood furniture can provide a long-lasting cooling effect and superior thermal comfort performance for use in summer and in areas with high average temperatures. However, the addition of powder had an effect on the gloss, hardness, and roughness of the paint film, so it is 
not advisable to add too much. Both $5 \%$ and $10 \%$ additions achieved a relatively good cooling effect, and the extinction phenomenon could be circumvented by adding PCM in layers. In actual production, adding $5 \%$ or $10 \%$ PCM to the primer and then superimposing the topcoat according to the process needs proved effective. This method can achieve the effect of regulating the temperature consciousness, but also can ensure the performance of the paint film.

\section{ACKNOWLEDGMENTS}

This work was supported by a Scientific Research Foundation of a Project funded by the Collaborative Innovation Center for Efficient Utilization of Forestry Resources in Jiangsu Province.

\section{REFERENCES CITED}

Alay, S., Alkan, C., and Göde, F. (2011). "Synthesis and characterization of poly (methyl methacrylate)/n-hexadecane microcapsules using different cross-linkers and their application to some fabrics," Thermochimica Acta 518(1-2), 1-8. DOI: 10.1016/j.tca.2011.01.014

Alkan, C., Sarı, A., and Karaipekli, A. (2011). "Preparation, thermal properties and thermal reliability of microencapsulated n-eicosane as novel phase change material for thermal energy storage," Energy Conversion and Management 52(1), 687-692. DOI: 10.1016/j.enconman.2010.07.047

Feldman, D., Banu, D., Hawes, D., and Ghanbari, E. (1991). “Obtaining an energy storing building material by direct incorporation of an organic phase change material in gypsum wallboard," Solar Energy Materials 22(2-3), 231-242. DOI: 10.1016/0165-1633(91)90021-C

Han, P., Lu, L., Qiu, X., Tang, Y., and Wang, J. (2015). "Preparation and characterization of macrocapsules containing microencapsulated PCMs (phase change materials) for thermal energy storage," Energy 91(C), 531-539. DOI: 10.1016/j.energy.2015.08.001

Huang, D. W., and Wang, H. M. (2012). "Phase change materials of microcapsules containing paraffin," Advanced Materials Research 482-484, 1596-1599. DOI: 10.4028/www.scientific.net/AMR.482-484.1596

Hunger, M., Entrop, A. G., Mandilaras, I., Brouwers, H. J. H., and Founti, M. (2009). "The behavior of self-compacting concrete containing micro-encapsulated phase change materials," Cement and Concrete Composites 31(10), 731-743. DOI: 10.1016/j.cemconcomp.2009.08.002

ISO 15184 (2012). "Paints and varnishes-Determination of film hardness by pencil test," International Organization for Standardization, Geneva, Switzerland.

ISO 2409 (2020). "Paints and varnishes - Cross-cut test," International Organization for Standardization, Geneva, Switzerland.

ISO 2813 (2014). "Paints and varnishes-Determination of gloss value at $20^{\circ}, 60^{\circ}$ and $85^{\circ}$," International Organization for Standardization, Geneva, Switzerland. 
Jeong, S., Jeon, J., Seo, J., Lee, J., and Kim, S. (2012). "Performance evaluation of the microencapsulated PCM for wood-based flooring application," Energy Conversion and Management 64, 516-521. DOI: 10.1016/j.enconman.2012.03.007

Khadiran, T., Hussein, M. Z., Zainal, Z., and Rusli, R. (2016). “Advanced energy storage materials for building applications and their thermal performance characterization: A review," Renewable \& Sustainable Energy Reviews 57, 916-928. DOI:

10.1016/j.rser.2015.12.081

Lan, L., Xia, L., Tang, J., Wyon, D. P., and Liu, H. (2019). "Mean skin temperature estimated from 3 measuring points can predict sleeping thermal sensation," Building and Environment 162, article ID 106292. DOI: 10.1016/j.buildenv.2019.106292

Maula, H., Hongisto, V., Östman, L., Haapakangas, A., Koskela, H., and Hyönä, J. (2016). "The effect of slightly warm temperature on work performance and comfort in open-plan offices - A laboratory study," Indoor Air 26(2), 286-297. DOI: 10.1111/ina.12209

Pasupathy, A., Velraj, R., and Seeniraj, R. V. (2008). "Phase change material-based building architecture for thermal management in residential and commercial establishments," Renewable and Sustainable Energy Reviews 12(1), 39-64. DOI: 10.1016/j.rser.2006.05.010

Peng, G., Dou, G., Hu, Y., Sun, Y., and Chen, Z. (2020). "Phase change material (PCM) microcapsules for thermal energy storage," Advances in Polymer Technology 2020(3), article ID 9490873. DOI: 10.1155/2020/9490873

Sari, A., Alkan, C., and Bilgin, C. (2014). "Micro/nano encapsulation of some paraffin eutectic mixtures with poly(methyl methacrylate) shell: Preparation, characterization and latent heat thermal energy storage properties," Applied Energy 136, 217-227. DOI: 10.1016/j.apenergy.2014.09.047

Tham, S., Thompson, R., Landeg, O., Murray, K. A., and Waite, T. (2020). "Indoor temperature and health: A global systematic review," Public Health 179, 9-17. DOI: 10.1016/j.puhe.2019.09.005

Wang, S., Lin, F., and Lin, M. (2000). "Thermal properties of interior decorative material and contacted sensory cold-warmth I: Relation between skin temperature and contacted sensory cold-warmth," Journal of wood science 46(5), 357-363. DOI: 10.1007/BF00776396

Xu, D., and Yang, R. (2019). "Efficient preparation and characterization of paraffinbased microcapsules by emulsion polymerization," Journal of Applied Polymer Science 136(21), article ID 47552. DOI: 10.1002/app.47552

Yan, X., and Chang, Y. (2019a). "Investigation of waterborne thermochromic topcoat film with color-changing microcapsules on Chinese fir surface," Progress in Organic Coatings 136(2), article ID 105262. DOI: 10.1016/j.porgcoat.2019.105262

Yan, X., Zhao, W., Wang, L., and Qian, X. (2021b). "Effect of microcapsule concentration with different core-shell ratios on waterborne topcoat film properties for Tilia europaea," Coatings 11(9), article number 1013. DOI: 10.3390/coatings 11091013

Yan, X., Peng, W., and Qian, X. (2021a). "Effect of water-based acrylic acid microcapsules on the properties of paint film for furniture surface," Applied Sciences 11(16), article number 7586. DOI: 10.3390/app11167586

Yan, X., Qian, X., and Chang, Y. (2019b). "Preparation and characterization of urea formaldehyde@ epoxy resin microcapsule on waterborne wood coatings," Coatings 9(8), article number 475. DOI: 10.3390/coatings9080475 
Yu, B., and Pei, K., (2020). "Preparation and properties of paraffin phase change microcapsules for temperature auto-adjusting coatings," Journal of Zhejiang Sci-Tech University (Natural Sciences Edition) 43(5), 602-608. DOI: 10.3969/j.issn.16733851(n).2020.05.003

Article submitted: November 18, 2021; Peer review completed: December 26, 2021;

Revised version received and accepted: December 29, 2021; Published: January 6, 2022.

DOI: 10.15376/biores.17.1.1319-1337 